Arch. Math. 87 (2006) 217-226

0003-889X/06/030217-10

DOI 10.1007/s00013-006-1139-6

(C) Birkhäuser Verlag, Basel, 2006

Archiv der Mathematik

\title{
On toric $h$-vectors of centrally symmetric polytopes
}

\author{
By \\ ANNETTE A'CAMPO-NEUEN
}

\begin{abstract}
We prove tight lower bounds for the coefficients of the toric $h$-vector of an arbitrary centrally symmetric polytope generalizing previous results due to R. Stanley and the author using toric varieties. Our proof here is based on the theory of combinatorial intersection cohomology for normal fans of polytopes developed by G. Barthel, J.-P. Brasselet, K. Fieseler and L. Kaup, and independently by P. Bressler and V. Lunts. This theory is also valid for nonrational polytopes when there is no standard correspondence with toric varieties. In this way we can establish our bounds for centrally symmetric polytopes even without requiring them to be rational.
\end{abstract}

Introduction. The $h$-vector of a simplicial polytope is defined as a linear combination of the face numbers of the polytope, and its entries have a topological interpretation as the Betti numbers of the quasi-smooth toric variety corresponding to the polytope. For the $h$-vector of a centrally symmetric simplicial polytope, R. Stanley proved tight lower bounds (see [10]) using the theory of toric varieties. His results were then generalized by R. Adin who considered simplicial polytopes with a certain symmetry of prime power order (see [3]).

For arbitrary polytopes, R. Stanley introduced the notion of a generalized h-vector (see [11]). This combinatorial invariant of the polytope is defined by recursion over its faces, and in the simplicial case it coincides with the usual $h$-vector. If the polytope $P$ is rational then there is an associated projective toric variety $X_{P}$, and the coefficients of the generalized $h$-vector of $P$ are the Betti numbers of the intersection cohomology of middle perversity of $X_{P}$. Because of this interpretation, the generalized $h$-vector is also referred to as the toric $h$-vector of the polytope (see [6]).

In a previous article, we considered rational polytopes with the type of symmetry investigated by Adin (see [1]). We asked for conditions imposed by the existence of the symmetry on the toric $h$-vector of the polytope. Using its topological interpretation via intersection cohomology of middle perversity, we obtained tight lower bounds for the coefficients of the toric $h$-vector in this situation. In the case of a centrally symmetric simplicial polytope, we get back the bounds due to Stanley (see [10]).

Mathematics Subject Classification (2000): 52B12, 52B05, 14M25, 14F43. 
The aim of this article is to show that the same bounds remain valid even if we do not assume the centrally symmetric polytope to be rational. Our proof is based on the theory of combinatorial intersection cohomology for fans developed by G. Barthel, J.-P. Brasselet, K. Fieseler and L. Kaup (see [5]) and independently by P. Bressler and V. Lunts (see [7]).

They discovered that one can completely characterize the intersection cohomology of middle perversity of a toric variety by combinatorial and algebraic data associated to the corresponding fan, namely in terms of a minimal extension sheaf on the fan considered as a topological space where the subfans are the open subsets (see [4]). Associating an analogous object to a non-rational fan, they define a combinatorial intersection cohomology satisfying similar formal properties as the usual intersection cohomology.

Both teams of authors conjectured that for the combinatorial intersection cohomology of a fan arising from a polytope, a combinatorial version of the Hard Lefschetz Theorem holds. Moreover, they proved that if such a Hard Lefschetz theorem is true then the even Betti numbers of the combinatorial intersection cohomology are precisely the coefficients of the toric $h$-vector of the corresponding polytope (see [5] and [7]).

The Hard Lefschetz Theorem in this context was recently proved by Kalle Karu (see [9]). The fact that a combinatorial Hard Lefschetz Theorem holds has striking consequences. For example, the coefficients of the toric $h$-vector of an arbitrary polytope are non-negative which is not at all clear from the definition. Moreover, the toric $h$-vector of an arbitrary polytope is unimodal.

We apply these results to a centrally symmetric polytope $P$ of dimension $n$. Denoting its toric $h$-vector by $\left(h_{0}, \ldots, h_{n}\right)$, we prove the following for the polynomial $h_{P}:=\sum_{j=0}^{n} h_{j} x^{j}$ (see Theorem 4.2):

Theorem. If a polytope $P$ of dimension $n$ admits a central symmetry then the polynomial

$$
h_{P}(x)-(1+x)^{n}
$$

has nonnegative, even coefficients, it is palindromic and unimodal. That means that we have the following bounds for the coefficients $h_{j}$ of $h_{P}$ :

$$
h_{j}-h_{j-1} \geqq\left(\begin{array}{c}
n \\
j
\end{array}\right)-\left(\begin{array}{c}
n \\
j-1
\end{array}\right) \quad \text { for } j=1, \ldots,\left[\frac{n}{2}\right] \text {. }
$$

Note that $(1+x)^{n}$ occurs as the $h$-polynomial of the $n$-dimensional cross-polytope. We can reformulate the lower bounds given by the theorem in terms of the partial ordering on real polynomials of degree $n$ defined by coefficientwise comparison, i.e., $a=\sum_{j=0}^{n} a_{j} x^{j} \leqq b=$ $\sum_{j=0}^{n} b_{j} x^{j}$ if and only if $a_{j} \leqq b_{j}$ for all $j$. The $h$-polynomial of the $n$-dimensional crosspolytope is minimal in this sense and in fact this is the only polytope realizing the minimum (see Corollary 4.3). 
Corollary. Let $P$ be an $n$-dimensional centrally symmetric polytope. Then

$$
h_{P} \geqq(1+x)^{n} .
$$

Moreover, equality holds if and only if the polytope $P$ is affinely equivalent to the n-dimensional cross-polytope.

1. Preliminaries. Let $P$ denote a convex polytope of dimension $n$ in $V:=\mathbb{R}^{n}$. Assume that the origin lies in the interior of $P$. Then the polytope $P$ defines a complete fan in $V$ consisting of the cones through its proper faces:

$$
\Delta_{P}:=\{\operatorname{cone}(F) ; F \text { proper face of } P\} \cup\{0\},
$$

Moreover, this fan is equipped with a strictly concave support function, that means a concave function whose restriction to any cone in $\Delta_{P}$ is linear and such that for any two different maximal cones the linear functions obtained by restriction are different. To define this function, consider the dual polytope $P^{*}:=\left\{u \in V^{*} ;\langle u, v\rangle \leqq-1\right.$ for all $\left.v \in P\right\}$ of $P$. There is an order-reversing one-to-one-correspondence between the proper faces of $P$ and the proper faces of $P^{*}$ defined by

$$
F \mapsto s_{F}:=\left\{u \in P^{*} ;\langle u, v\rangle=-1 \text { for all } v \in F\right\}
$$

So the vertices of $P^{*}$ are of the form $s_{F}$, where $F$ is a one-codimensional face of $P$, and $s_{F}$ defines a linear function on cone $(F)$. These linear functions glue together to a well-defined concave function $s_{P}: V \rightarrow \mathbb{R}$.

The toric $h$-vector of the polytope $P$ is a combinatorial invariant defined by recursion over the faces of $P$ (see [11]). In fact, this invariant only depends on the fan $\Delta_{P}$, and it makes sense to define a toric $h$-vector for arbitrary complete fans using the same recursion formulae. So let us state the definition here in terms of fans. A fan in a real vector space $V$ is a nonempty set $\Delta$ of strictly convex polyhedral cones intersecting pairwise in common faces and such that if a cone belongs to the set $\Delta$ then all its faces also belong to $\Delta$. The fan $\Delta$ is called complete if its support $|\Delta|:=\bigcup_{\sigma \in \Delta} \sigma$ equals $V$, and $\Delta$ is rational with respect to a lattice $N$ in $\mathbb{R}^{n}$, if all the cones are generated by vectors in $N$. For a given cone $\sigma$, let $V_{\sigma}$ denote the linear span of $\sigma$ in $V$. Let $\Lambda_{\sigma}$ denote the fan that we obtain by projecting the boundary of $\sigma$ to $V_{\sigma} / L$, where $L$ is a one-dimensional subspace generated by a vector in the relative interior of $\sigma$.

We introduce two polynomials, namely $h_{\Delta}$ for each complete fan $\Delta$ and $g_{\sigma}$ for each strictly convex polyhedral cone $\sigma$, satisfying the following recursion:

i) $g_{0} \equiv 1$,

ii) $h_{\Delta}(x)=\sum_{\sigma \in \Delta}(x-1)^{\operatorname{dim} \Delta-\operatorname{dim} \sigma} g_{\sigma}(x)$,

iii) $g_{\sigma}(x)=\tau_{\leqq[(\operatorname{dim} \sigma) / 2]}\left((1-x) h_{\Lambda_{\sigma}}(x)\right)$, 
where $\tau_{\leqq}$denotes the truncation operator $\tau_{\leqq}\left(\sum_{i=0}^{n} a_{i} x^{i}\right):=\sum_{i=0}^{r} a_{i} x^{i}$. So by definition, the coefficients of the polynomial $g_{\sigma}$ are differences of successive coefficients of the polynomial $h_{\Lambda_{\sigma}}$.

The vector formed by the coefficients of the polynomial $h_{\Delta}$ is called the toric $h$-vector of the fan $\Delta$. For a polytope $P$, we set $h_{P}:=h_{\Delta_{P}}$.

For example $h_{n}=1$ and $h_{n-1}=|\{\sigma \in \Delta ; \operatorname{dim} \sigma=1\}|-n$, where $n$ denotes the dimension of the fan.

If $\sigma$ is a simplicial cone, then $g_{\sigma}=1$. Hence if $\Delta=\Delta_{P}$ is the fan through the faces of a simplicial polytope $P$, then $h_{P}=h_{\Delta}=\sum_{\sigma \in \Delta}(x-1)^{\operatorname{dim} \Delta-\operatorname{dim} \sigma}$, which is nothing but the usual $h$-vector. But for a general polytope, its toric $h$-vector does not only depend on the face numbers but is more complicated.

Stanley showed that the toric $h$-vector of an $n$-dimensional polytope is palindromic, that means that $h_{j}=h_{n-j}$ for all $j$. To give an example, the toric $h$-vector of the 3-dimensional cross-polytope is $(1,3,3,1)$ and the toric $h$-vector of its dual, the 3 -dimensional cube, is $(1,5,5,1)$.

2. Combinatorial Intersection Cohomology. For later use, in this section we very briefly summarize some results on combinatorial intersection cohomology that are presented in [5] and [7]. We also formulate the hard Lefschetz theorem in this context proved in [9]. We will follow the notation used in [5]. Let $\Delta$ be a (not necessarily rational) fan in a real vectorspace $V=\mathbb{R}^{n}$.

If a subset of cones $\Lambda \subset \Delta$ is again a fan, then we speak of a subfan and write $\Lambda \prec \Delta$. In the rational case, where $\Delta$ defines a toric variety, the subfans of $\Delta$ correspond to the open invariant subsets of the toric variety, so they define a " $T$-stable topology". That is the motivation for considering the set of all subfans of an arbitrary fan $\Delta$ together with the empty set as the open sets of a topology, namely the fan topology on $\Delta$. A basis of this topology is formed by the affine subfans, i.e., the subfans that are fans of faces of single cones. For a cone $\sigma \in \Delta$, we denote the fan of faces of $\sigma$ by $\langle\sigma\rangle$ and its boundary fan by $\partial \sigma$.

Let $A^{\bullet}:=S^{\bullet}\left(V^{*}\right)$ denote the algebra of real-valued polynomial functions on $V$, together with the grading defined by associating to each linear function the degree 2 . The algebra $A^{\bullet}$ defines a sheaf of graded algebras $\mathcal{A}^{\bullet}$ on $\Delta$ (with the fan topology), where for $\sigma \in \Delta$ the algebra $\mathcal{A}^{\bullet}(\langle\sigma\rangle)=$ : $A_{\sigma}^{\bullet}$ consists of the elements of $S^{\bullet}\left(V_{\sigma}^{*}\right)$ viewed as polynomial functions on $\sigma$. The restriction homomorphisms of $\mathcal{A}^{\bullet}$ are given by restriction of polynomial functions. For $\Lambda \prec \Delta$, the sections in $\mathcal{A}^{\bullet}(\Lambda)$ correspond to those functions on $\Lambda$ that are conewise polynomial. Instead of $\mathcal{A}^{\bullet}(\Lambda)$ we also write $A_{\Lambda}^{\bullet}$.

Now consider a sheaf $\mathcal{E}^{\bullet}$ of graded $\mathcal{A}^{\bullet}$-modules on $\Delta$. To denote the sections $\mathcal{E}^{\bullet}(\Lambda)$ of $\mathcal{E}^{\bullet}$ on $\Lambda \prec \Delta$ we also write $E_{\Lambda}$, and we abbreviate $E_{\langle\sigma\rangle}$ to $E_{\sigma}$. With $\bar{E}_{\Lambda}^{\bullet}$ we denote the graded real vector space obtained from the graded $A_{\Lambda}^{\bullet}$-module $E_{\Lambda}^{\bullet}$ by taking residue classes modulo the unique homogeneous maximal ideal of $A_{\Lambda}^{\bullet}$.

The sheaf $\mathcal{E}^{\bullet}$ is called a minimal extension sheaf if the following properties hold: 
i) $E_{0}^{\bullet} \simeq \mathbb{R}^{\bullet}$, where $\mathbb{R}^{\bullet}$ denotes $\mathbb{R}$ viewed as a graded algebra with trivial zero grading.

ii) For every $\sigma \in \Delta$, the module $E_{\sigma}^{\bullet}$ is free over $A_{\sigma}^{\bullet}$.

iii) For each cone $\sigma \in \Delta \backslash\{0\}$, the restriction map $\rho_{\sigma}: E_{\sigma}^{\bullet} \rightarrow E_{\partial \sigma}^{\bullet}$ induces an isomorphism

$$
\bar{\rho}_{\sigma}: \bar{E}_{\sigma}^{\bullet} \rightarrow \bar{E}_{\partial \sigma}^{\bullet}
$$

of graded real vector spaces.

In [5] and [7], the authors prove that for any given fan $\Delta$, a minimal extension sheaf exists and is unique up to isomorphism.

If the fan is rational, then we have an associated toric variety $X_{\Delta}$. The equivariant intersection cohomology of open subsets of $X_{\Delta}$ defines a minimal extension sheaf on $\Delta$ by the assignment $\Lambda \mapsto I H_{T}^{*}\left(X_{\Lambda} ; \mathbb{R}\right)$ for $\Lambda \prec \Delta$, so in particular $E_{\Delta}^{\bullet} \simeq I H_{T}^{*}\left(X_{\Delta} ; \mathbb{R}\right)$. Moreover, $\bar{E}_{\Delta}^{\bullet}$ is isomorphic to the intersection cohomology of middle perversity $I H^{*}\left(X_{\Delta} ; \mathbb{R}\right)$ of $X_{\Delta}$ (see [4]).

If the fan $\Delta$ is not rational, then there is no way of associating a toric variety to it. But the construction of the sheaf $\mathcal{E}^{\bullet}$ still makes sense, and the authors define $I H^{\bullet}(\Delta):={\overline{E_{\Delta}}}_{\Delta}$ to be the combinatorial intersection cohomology of $\Delta$.

Now let us assume that $\Delta=\Delta_{P}$ arises from a polytope P. Then the following holds (see [5] and [7]):

Theorem. 2.1 (Barthel, Brasselet, Fieseler, Kaup; Bressler, Lunts). $E_{\Delta}^{\bullet}$ is a free $A^{\bullet}$-module, and therefore $E_{\Delta}^{\bullet}=A^{\bullet} \otimes_{\mathbb{R}} \bar{E}_{\Delta}^{\bullet}$.

Moreover, the following analogue of the hard Lefschetz theorem was conjectured in [5] and [7] and then proved by K. Karu (see [9]):

Theorem 2.2 (Karu). Let $s_{P}$ be the strictly concave support function on $\Delta=\Delta_{P}$ defined by the polytope $P$. Then the map $\bar{\mu}^{2 q}: \bar{E}_{\Delta}^{2 q} \rightarrow \bar{E}_{\Delta}^{2 q+2}$ induced by the multiplication with $s_{P} \in \mathcal{A}^{2}(\Delta)$ is injective for $2 q \leqq n-1$ and surjective for $2 q \geqq n-1$.

Assuming the hard Lefschetz theorem, it is proved in [5] and [7] that the Betti-numbers of the combinatorial intersection cohomology are precisely given by the coefficients of the toric $h$-vector of the fan.

Theorem 2.3 (Barthel, Brasselet, Fieseler, Kaup; Bressler, Lunts; Karu).

$$
h_{\Delta}\left(t^{2}\right)=\sum_{q=0}^{2 n}\left(\operatorname{dim} \bar{E}_{\Delta}^{q}\right) t^{q} .
$$

The fact that the $h$-polynomial is palindromic is reflected by a combinatorial version of Poincaré-duality, also proved in [5] and [7]. 
We now define a Poincaré-series $v_{\Delta}$ for the graded algebra $E_{\Delta}^{\bullet}$ and a Poincaré-polynomial $u_{\Delta}$ for the graded vector space $\bar{E}_{\Delta}^{\bullet}$ as follows:

$$
v_{\Delta}(t):=\sum_{q \geqq 0}\left(\operatorname{dim} E_{\Delta}^{q}\right) t^{q} \quad \text { and } \quad u_{\Delta}(t):=\sum_{q \geqq 0}\left(\operatorname{dim} \bar{E}_{\Delta}^{q}\right) t^{q} .
$$

Note that here we differ slightly from the convention used in [5] in order to be consistent with the notation used in [1]. One obtains the Poincaré-series used in [5] from ours by viewing it as a function in $t^{2}$.

As a consequence of the first part of the above theorem we obtain:

$$
v_{\Delta}(t)=\frac{1}{\left(1-t^{2}\right)^{n}} \cdot u_{\Delta}(t)
$$

3. Refined Poincaré-series. From now on let $\Delta$ denote a complete fan, and assume that for every $\sigma \in \Delta$ also $-\sigma \in \Delta$, in other words assume that $\Delta$ is centrally symmetric. Let $\varphi=-\mathrm{id}_{V}$ denote the central symmetry. Being an invertible linear transformation, $\varphi$ induces an $\mathbb{R}$-linear automorphism of the graded algebra $A^{\bullet}=S^{\bullet}\left(V^{*}\right)$. Note that for every cone $\sigma \in \Delta$, we have $V_{\sigma}=V_{-\sigma}$. Since $A_{\sigma}^{\bullet}$ is the algebra of polynomial functions on $V_{\sigma}$ restricted to $\sigma$, the algebras $A_{\sigma}^{\bullet}$ and $A_{-\sigma}^{\bullet}$ are not identical, but canonically isomorphic. The action of $\varphi$ on $V_{\sigma}$ induces an isomorphism of graded algebras from $A_{\sigma}^{\bullet}$ to $A_{-\sigma}^{\bullet}$ that is compatible with this canonical isomorphism. Moreover, the induced isomorphisms are compatible with the restriction homomorphisms $\rho_{\tau}^{\sigma}: A_{\sigma}^{\bullet} \rightarrow A_{\tau}^{\bullet}$ for every $\tau \prec \sigma$. That means that in fact $\varphi$ defines a natural automorphism of $\Delta$ as a ringed space equipped with the sheaf of graded algebras $\mathcal{A}^{\bullet}$. We can also define an action of $\varphi$ on the minimal extension sheaf $\mathcal{E}^{\bullet}$ on $\Delta$.

\section{Lemma 3.1. There are isomorphisms of graded vector spaces}

$$
\varphi: E_{\sigma}^{\bullet} \rightarrow E_{-\sigma}^{\bullet}
$$

for every $\sigma \in \Delta$ that are equivariant with respect to the module structure over $A_{\sigma}^{\bullet}$ and $A_{-\sigma}^{\bullet}$ respectively and compatible with the restriction homomorphisms of the sheaf $\mathcal{E}^{\bullet}$.

P r o of. To define the required isomorphisms, we proceed by recursion over the $k$-skeleton $\Delta^{\leq k}$ of $\Delta$ following the recursive construction of $\mathcal{E}^{\bullet}$ as in Section 1 of [5]. On $E_{0}^{\bullet}=\mathbb{R}^{\bullet}$, where 0 denotes the zero cone, $\varphi$ acts as the identity. Now assume that the isomorphisms have been defined for $\Delta^{<k}$, and let $\sigma \in \Delta^{k}$. We can assume that $E_{\sigma}^{\bullet}=A_{\sigma}^{\bullet} \otimes_{\mathbb{R}} \bar{E}_{\partial \sigma}^{\bullet}$. By induction, we already have an isomorphism $\varphi: E_{\partial \sigma}^{\bullet} \rightarrow E_{-\partial \sigma}^{\bullet}$, and since the maximal ideal $\mathfrak{m}$ of $\mathcal{A}^{\bullet}$ is $\varphi$-stable, $\varphi$ induces an isomorphism $\bar{\varphi}: \bar{E}_{\partial \sigma}^{\bullet} \rightarrow \bar{E}_{-\partial \sigma}^{\bullet}$. Together with the map from $A_{\sigma}^{\bullet}$ to $A_{-\sigma}^{\bullet}$ determined by $\varphi$, that provides us with an isomorphism of graded vector spaces $\varphi: E_{\sigma}^{\bullet} \rightarrow E_{-\sigma}^{\bullet}$. By construction, this map is equivariant as a map from an $A_{\sigma}^{\bullet}$-module to an $A_{-\sigma}^{\bullet}$-module.

In the construction of $\mathcal{E}^{\bullet}$, the restriction homomorphism from $E_{\sigma}^{\bullet}=A_{\sigma}^{\bullet} \otimes_{\mathbb{R}} \bar{E}_{\partial \sigma}^{\bullet}$ to $E_{\partial \sigma}^{\bullet}$ is defined using the restriction homomorphism $\rho_{\partial \sigma}^{\sigma}: A_{\sigma}^{\bullet} \rightarrow A_{\partial \sigma}^{\bullet}$ and an $\mathbb{R}$-linear section 
$s_{\sigma}: \bar{E}_{\partial \sigma}^{\bullet} \rightarrow E_{\partial \sigma}^{\bullet}$ of the residue class map $E_{\partial \sigma}^{\bullet} \rightarrow \bar{E}_{\partial \sigma}^{\bullet}$. The section $s_{\sigma}$ can be chosen freely. So we can assume that for any pair $\sigma,-\sigma$ of antipodal cones in $\Delta$ the corresponding sections have been chosen such that the following diagram is commutative:

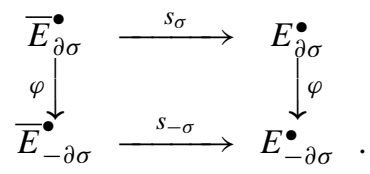

That implies compatibility of $\varphi$ with the restriction homomorphisms of $\mathcal{E}^{\bullet}$.

So we have an automorphism $\varphi$ of the sheaf $\mathcal{E}^{\bullet}$. This automorphism is in fact canonical, i.e., it is uniquely determined by the given action of $\varphi$ on $\Delta$. That follows from Remark 1.8 (iii) in [5] together with Theorem 2.2.

We also obtain an induced automorphism $\varphi$ on the module $E_{\Delta}^{\bullet}$ of global sections of $\mathcal{E}^{\bullet}$. Each graded piece $E_{\Delta}^{q}$ of $E_{\Delta}^{\bullet}$ decomposes into eigenspaces under the action of $\varphi$. Let us denote the eigenspaces for the eigenvalues +1 and -1 by $\left(E_{\Delta}^{q}\right)^{+}$and $\left(E_{\Delta}^{q}\right)^{-}$respectively.

The so-called refined Poincaré-series for the action of $\varphi$ on $E_{\Delta}^{\bullet}$ is defined as the following series over the group ring $\mathbb{Z}[G]$ of the character group $G:=\{ \pm 1\}$ of the group generated by $\varphi$ in $\mathrm{GL}(V)$, namely:

$$
v_{\Delta}^{\varphi}(t):=\sum_{q \geqslant 0}\left(\operatorname{dim}\left(E_{\Delta}^{q}\right)^{+}+\operatorname{dim}\left(E_{\Delta}^{q}\right)^{-} \chi\right) t^{q},
$$

where $\chi$ denotes the element corresponding to -1 in $\mathbb{Z}[G]$. The refined Poincaré-polynomial $u_{\Delta}^{\varphi}$ for the action of $\varphi$ on $\bar{E}_{\Delta}^{\bullet}$ is defined analogously.

By Theorem 2.1, $E_{\Delta}^{\bullet}$ is a free $A^{\bullet}$-module. The action of $\varphi$ on $\bar{E}_{\Delta}^{\bullet}$ is induced by taking residue classes. So if we choose a homogeneous basis for $\bar{E}_{\Delta}^{\bullet}$ and preimages under the residue class map in $E_{\Delta}^{\bullet}$ to define an isomorphism

$$
E_{\Delta}^{\bullet} \rightarrow \bar{E}_{\Delta}^{\bullet} \otimes_{\mathbb{R}} A^{\bullet},
$$

then this isomorphism is automatically compatible with the action of $\varphi$. That implies

$$
v_{\Delta}^{\varphi}(t)=\frac{1}{\left(1-\chi t^{2}\right)^{n}} \cdot u_{\Delta}^{\varphi}(t) .
$$

To obtain a relation between the Poincaré-series $v_{\Delta}$ and its refined version $v_{\Delta}^{\varphi}$, we can use the fact that the minimal extension sheaf $\mathcal{E}^{\bullet}$ as a sheaf of real vector spaces can be written as a direct sum of simpler subsheafs. Note that $\mathcal{E}^{\bullet}$ is a flabby sheaf on $\Delta$. Here that means that the restriction homomorphism $\rho_{\partial \sigma}^{\sigma}: E_{\sigma}^{\bullet} \rightarrow E_{\partial \sigma}^{\bullet}$ is surjective for all $\sigma \in \Delta$.

For $\sigma \in \Delta$, let $\mathcal{J}_{\sigma}$ denote the characteristic sheaf of $\sigma$ defined on $\Lambda \prec \Delta$ by

$$
\mathcal{J}_{\sigma}(\Lambda):= \begin{cases}\mathbb{R} & \text { if } \sigma \in \Lambda \\ 0 & \text { otherwise }\end{cases}
$$


Then there is an isomorphism of sheafs of graded real vector spaces

$$
\mathcal{E}^{\bullet} \simeq \bigoplus_{\sigma \in \Delta} \mathcal{J}_{\sigma} \otimes_{\mathbb{R}} K_{\sigma}
$$

where $K_{\sigma}$ denotes the kernel of the restriction homomorphism $\rho_{\partial \sigma}^{\sigma}: E_{\sigma}^{\bullet} \rightarrow E_{\partial \sigma}^{\bullet}$ (see [5, Section 3]).

For every $\sigma \in \Delta, \varphi$ induces a map from $\mathcal{J}_{\sigma}$ to $\mathcal{J}_{-\sigma}$ that is compatible with the action of $\varphi$ on $\Delta$. And using these maps together with the action of $\varphi$ on $\mathcal{E}^{\bullet}$, we obtain an induced $\varphi$-action on the direct sum on the righthandside of (3), where $\varphi$ maps $\mathcal{J}_{\sigma}$ to $\mathcal{J}_{-\sigma}$ and $K_{\sigma}$ to $K_{-\sigma}$.

Since the automorphism of $\mathcal{E}^{\bullet}$ induced by $\varphi$ is canonical, the actions of $\varphi$ on both sides of (3) must be compatible. So in fact the isomorphism (3) is $\varphi$-equivariant.

Now consider the action of $\varphi$ on the direct sum on the righthandside of (3). Apparently, for every cone $\sigma \neq 0$, the action of $\varphi$ interchanges the summands $\mathcal{J}_{\sigma} \otimes_{\mathbb{R}} K_{\sigma}$ and $\mathcal{J}_{-\sigma} \otimes_{\mathbb{R}} K_{-\sigma}$. So for every $q$, in $\bigoplus_{\sigma \neq 0} \mathcal{J}_{\sigma} \otimes_{\mathbb{R}} K_{\sigma}$ the eigenvalue +1 and -1 occur with the same multiplicity.

We obtain the relation

$$
v_{\Delta}^{\varphi}(t)-1=\frac{1}{2}\left(\left(v_{\Delta}(t)-1\right)+\left(v_{\Delta}(t)-1\right) \cdot \chi\right)=\frac{1}{2}(1+\chi)\left(v_{\Delta}(t)-1\right) .
$$

4. Lower bounds for the generalized $\boldsymbol{h}$-vector. Summarizing the considerations in the previous section, we obtain the following description of the refined Poincaré-polynomial:

Proposition 4.1. Let $\Delta$ be a centrally symmetric complete fan of dimension $n$. Then

$$
u_{\Delta}^{\varphi}(t)=\frac{1}{2}\left(u_{\Delta}(t)+\left(1+t^{2}\right)^{n}\right)+\frac{1}{2} \chi\left(u_{\Delta}(t)-\left(1+t^{2}\right)^{n}\right) .
$$

P r o o f. Inserting (1) in (4), we obtain

$$
v_{\Delta}^{\varphi}(t)=\frac{1}{2}(1+\chi) v_{\Delta}(t)+\frac{1-\chi}{2} .
$$

Using (2), that implies

$$
u_{\Delta}^{\varphi}(t)=\frac{1}{2} \frac{(1+\chi)\left(1-\chi t^{2}\right)^{n}}{\left(1-t^{2}\right)^{n}} u_{\Delta}(t)+\frac{1-\chi}{2}\left(1-\chi t^{2}\right)^{n} .
$$

Note that since $\chi^{2}=1$, we have $\left(1-\chi t^{2}\right)(1+\chi)=\left(1-t^{2}\right)(1+\chi)$ and $\left(1-\chi t^{2}\right)(1-\chi)$ $=\left(1+t^{2}\right)(1-\chi)$. This implies

$$
u_{\Delta}^{\varphi}(t)=\frac{1-\chi}{2}\left(1+t^{2}\right)^{n}+\frac{1+\chi}{2} u_{\Delta}(t) .
$$

We now apply this proposition to polytopal centrally symmetric fans. 
Theorem 4.2. Let $P$ be a centrally-symmetric polytope of dimension $n$. Then the polynomial

$$
h_{P}(x)-(1+x)^{n},
$$

has nonnegative, even coefficients, it is palindromic and unimodal. In particular, we have the following bounds for the coefficients $h_{j}$ of $h_{P}$ :

$$
h_{j}-h_{j-1} \geqq\left(\begin{array}{c}
n \\
j
\end{array}\right)-\left(\begin{array}{c}
n \\
j-1
\end{array}\right) \text { for } j=1, \ldots,\left[\frac{n}{2}\right] .
$$

P r o o f. As before let $\Delta:=\Delta_{P}$ denote the fan through the faces of $P$ and let $s_{P}$ denote the $\Delta$-strictly convex support function defined by $P$. By Theorem 2.3, $u_{\Delta}(t)=h_{P}\left(t^{2}\right)$. The symmetry follows immediately from the combinatorial Poincaré-duality. Moreover, by the above proposition, we have

$$
\frac{1}{2}\left(u_{\Delta}(t)-\left(1+t^{2}\right)^{n}\right)=\sum_{q \geqq 0}\left(\operatorname{dim}\left(\bar{E}_{\Delta}^{q}\right)^{-}\right) t^{q} .
$$

This implies in particular, that all the coefficients of $p(t):=u_{\Delta}(t)-\left(1+t^{2}\right)^{n}$ are nonnegative and even.

Since the support function $s_{P} \in \mathcal{A}^{2}(\Delta)$ is invariant under $\varphi$, we have

$$
\left.s_{P} \cdot\left(\bar{E}_{\Delta}^{q}\right)^{-} \subset \bar{E}_{\Delta}^{q+2}\right)^{-} .
$$

Now it follows from the combinatorial hard Lefschetz theorem (see Theorem 2.2) that $\operatorname{dim}\left(\left(\bar{E}_{\Delta}^{2 q}\right)^{-}\right) \leqq \operatorname{dim}\left(\left(\bar{E}_{\Delta}^{2 q+2}\right)^{-}\right)$for $2 q \leqq n-1$, and that means that the polynomial $p$ is unimodal.

Note that $(1+x)^{n}$ occurs as the $h$-polynomial of the $n$-dimensional cross-polytope. We can reformulate the lower bounds given by the theorem in terms of the partial ordering on real polynomials of degree $n$ defined by coefficientwise comparison, i.e., $a=\sum_{j=0}^{n} a_{j} x^{j} \leqq b=$ $\sum_{j=0}^{n} b_{j} x^{j}$ if and only if $a_{j} \leqq b_{j}$ for all $j$. The $h$-polynomial of the $n$-dimensional crosspolytope is minimal in this sense and in fact this is the only polytope realizing the minimum.

Corollary 4.3. Let $P$ be an n-dimensional centrally symmetric polytope. Then

$$
h_{P} \geqq(1+x)^{n} .
$$

Moreover, equality holds if and only if the polytope $P$ is affinely equivalent to the n-dimensional cross-polytope.

P r o o f. Suppose that $h_{P}=(1+x)^{n}$ for a centrally symmetric polytope $P$. Then in particular, $h_{n-1}=n$. On the other hand, as mentioned in Section 1, we always have, 
$h_{n-1}=\mid\{$ vertices of $P\} \mid-n$. So $P$ has $2 n$ vertices. Let us choose a facet $F$ of $P$. Since $F$ contains at least $n$ vertices, and it is disjoint from its opposite facet $-F$, we obtain that $P$ is the convex hull of $F$ and $-F$. That means that $P$ is affinely equivalent to the $n$-dimensional cross-polytope.

\section{References}

[1] A. A'CAMPO-NEUEN, On generalized $h$-vectors of rational polytopes with a symmetry of prime order. Discrete Comput. Geom. 22, 259-268 (1999).

[2] R. M. AdIN, On h-vectors and symmetry. Contemp. Math. 178, 1-20 (1994).

[3] R. M. ADIN, On face numbers of rational simplicial polytopes with symmetry. Adv. Math. 115, 269-285 (1995).

[4] G. BARTHEL, J.-P. BRASSELET, K.-H. FIESELER and L. KAUP, Equivariant intersection cohomology of toric varieties. In: Algebraic Geometry: Hirzebruch 70. Contemp. Math. AMS 241, 45-68 (1999).

[5] G. BARThEL, J.-P. BRASSELET, K.-H. FIESELER and L. KAUP, Combinatorial intersection cohomology for fans. Tôhoku Math. J. 54, 1-41 (2002).

[6] M. BAYER and R. EHRENBORG, The toric h-vectors of partially ordered sets. Transactions of the AMS 352(10), 4515-4531 (2000)

[7] P. BRESSLER and V. A. LUNTS, Intersection cohomology on nonrational polytopes. Compos. Math. 135(3), 245-278 (2003).

[8] K.-H. FIESELER, Rational intersection cohomology of projective toric varieties. J. reine angew. Math. 413, 88-98 (1991).

[9] K. KARU, Hard Lefschetz theorem for nonrational polytopes. Invent. math. 157(2), 419-447 (2004).

[10] R. StAnLEY, On the number of faces of centrally-symmetric simplicial polytopes. Graphs Combin. 3, 55-66 (1987).

[11] R. STANLEY, Generalized $h$-vectors, intersection cohomology of toric varieties, and related results. In: Commutative Algebra and Combinatorics (M. Nagata and H. Matsumura, eds.), Advanced Studies in Pure Math. 11, Kinokuniya, Tokyo, and North-Holland, Amsterdam/New York 187-213 (1987).

Received: 24 March 2004

Annette A'Campo-Neuen

Mathematisches Institut

Universität Basel

Rheinsprung 21

CH-4051 Basel

Switzerland

Annette.ACampo@unibas.ch 